\title{
Impact of Lygus hesperus (Hemiptera: Miridae) Feeding and Oviposition on Lettuce
}

\author{
Shimat V. Joseph ${ }^{\dagger}$ \\ University of California Cooperative Extension Monterey County, Salinas, CA 93901
}

Accepted for publication 6 August 2019.

\begin{abstract}
The western tarnished plant bug, Lygus hesperus Knight (Hemiptera: Miridae), is an important insect pest of lettuce, Lactuca sativa L. in the central coast region of California. However, little is documented on the injury symptoms relative to levels of $L$. hesperus infestation on lettuce. Experiments were conducted in which lettuce plants were exposed to $0,1,3,5$, and $10 \mathrm{~L}$. hesperus adults for 2 and 7 days. The major injury symptom observed was lesions on the midrib of the leaves, and under low to moderate infestation (e.g., one to five adults per plant) after 2 days of exposure these lesions were not significantly different from the insect-free control. Under heavy infestation (e.g., 10 adults per plant), however, lesions developed rather quickly after 2 days of
\end{abstract}

Abstract

The western tarnished plant bug, Lygus hesperus Knight (Hemiptera: Miridae), is a sporadic but serious pest of lettuce, Lactuca sativa L. (Asteraceae), in the central coast region of California. Lettuce was valued at more than US $\$ 1.3$ billion in Monterey County, CA, alone (Monterey County Agricultural Commissioner 2017). L. hesperus are found in both young and mature lettuce plants in the production fields (S. V. Joseph, unpublished data), causing economic losses worth several thousands of U.S. dollars each year in a typical 6-ha field. Management of this pest becomes extremely challenging in certain growing seasons when populations reach problematic levels.

Feeding injury caused by $L$. hesperus has been documented on vegetables (Fye 1984; Hill 1932, 1933; Jacobson 2002; Joseph et al. 2016; Varis 1978) but not clearly documented on lettuce. In the field, lesions or holes on midribs of lettuce leaves are often observed, and they are attributed to L. hesperus feeding. Any such damage detected during preharvest inspection could result in rejection of the affected lot. L. hesperus has piercing-sucking mouthparts that consist of four stylets in a proboscis. These stylets are used to probe host plants and feed on plant fluids. These stylets support preoral digestion of plant tissue through a steady flow of salivary enzymes (Backus et al. 2005; Miles 1972; Strong 1970). This feeding method is referred as "macerate-and-flush," and it causes necrotic tissue on the site of insertion (McPherson 2018). However, L. hesperus feeding injury is often difficult to confirm,

${ }^{\dagger}$ Corresponding author: S. V. Joseph; E-mail: svjoseph@uga.edu

Current address: University of Georgia, 1109 Experiment Street, Griffin, GA 30223.

The author(s) declare no conflict of interest.

C 2019 The American Phytopathological Society exposure. The number of lesions was positively associated with numbers of feeding sites and eggs. When heavy infestation lasted for a week, most of the plants died. At low to moderate infestation, more lesions developed compared with the insect-free control. After a weeklong exposure, an increase in number of lesions was negatively associated with plant height. The results emphasize the need for regular scouting to facilitate immediate management decisions and actions to reduce losses from $L$. hesperus feeding and oviposition injury in lettuce.

Keywords: entomology, western tarnished plant bug, Lactuca sativa, plant injury because no feeding sheath is left behind after stylet insertion (S. V. Joseph, personal observations).

Before L. hesperus moves into managed fields, it develops on weedy hosts such as wild radish (Raphanus raphanistrum L.), common groundsel (Senecio vulgaris L.), lupines (Lupinus spp.), milk thistle (Silybum marianum [L.] Gaertn.), and mustards (Brassica spp.) found adjacent to fields and in unmanaged, nonproduction zones (Zalom et al. 2018). L. hesperus adults move to lettuce when weed hosts senesce or stop producing flowers. Management of $L$. hesperus on lettuce primarily involves use of pyrethroids and a carbamate: methomyl (Joseph et al. 2017; Natwick et al. 2017). Precise, early diagnosis of L. hesperus injury in lettuce and understanding the development of injury are critical to determine the need for insecticide use and application timing to reduce damage. Thus, the major objectives of this study were to (i) validate the injury caused by L. hesperus on lettuce and (ii) document the development of injury symptoms through feeding and oviposition when lettuce plants are exposed to various densities of adult $L$. hesperus for short and long periods.

\section{Experiments and Statistical Analysis}

Experiments were conducted at the University of California Cooperative Extension Monterey County greenhouse in Salinas, CA, in 2016. Lettuce 'Spyglass' seeds were planted in 3.78-liter plastic pots filled with potting medium (Sunshine Aggregate Plus Mix 4, Sun Gro, Agawam, MA) and placed on a greenhouse bench. The plants were irrigated at 2 days schedule; because the plants were maintained for short intervals, fertilizer was not added.

L. hesperus adults were field-collected within $24 \mathrm{~h}$ of the start of each experiment from weed hosts surrounding production fields in Salinas and Chualar, CA, and were maintained in a collapsible mesh cage (catalog no. 1466AV, BioQuip, Rancho Dominguez, CA). In 
the current study, cylindrical cages were used as test arenas in the experiments. The details of the cylindrical cage (Fig. 1) are described in Joseph et al. (2016). The cage was formed by rolling a rectangular section of clear film (Grafix, Maple Heights, $\mathrm{OH}$ ) and attaching a no-see-um mesh fabric (catalog no. 7250NSW, BioQuip) on the top of the cylinder. After introducing L. hesperus adults on a potted lettuce plant, the plant was covered by inserting the open end of the cage up to $2.5 \mathrm{~cm}$ into the soil (Fig. 1). Lettuce plants were 4 weeks old when $L$. hesperus adults were introduced. Plants in all replicates were the same age.

L. hesperus adults were caged at densities of $0,1,3,5$, and 10 adults (male or female) per pot. The ratio of males to females in the sampled populations was approximately 50:50 based on a preliminary survey. The caged lettuce plants were exposed to $L$. hesperus for 2 and 7 days. Each exposure period ( 2 and 7 days) was evaluated separately. Treatments were replicated 10 times and arranged in a completely randomized design. After the exposure period, plants were uprooted, placed in plastic bags, and stored at $4^{\circ} \mathrm{C}$ for evaluation. Experiments were repeated twice with a total of 2 exposure periods ( 2 and 7 days $) \times 5$ density treatments $\times 10$ replications $\times 2$ experimental repetitions: 200 lettuce plants were exposed to $L$. hesperus. The experiments were repeated at 2-week intervals. All evaluations were completed within 2 days of termination of exposure periods. All live L. hesperus adults were recovered at the end of experiments. The collected plants were thoroughly examined for $L$. hesperus feeding and oviposition injury using a dissecting microscope at $10 \times$ magnification. The number of discolored spots (hereafter referred as "feeding sites") related to

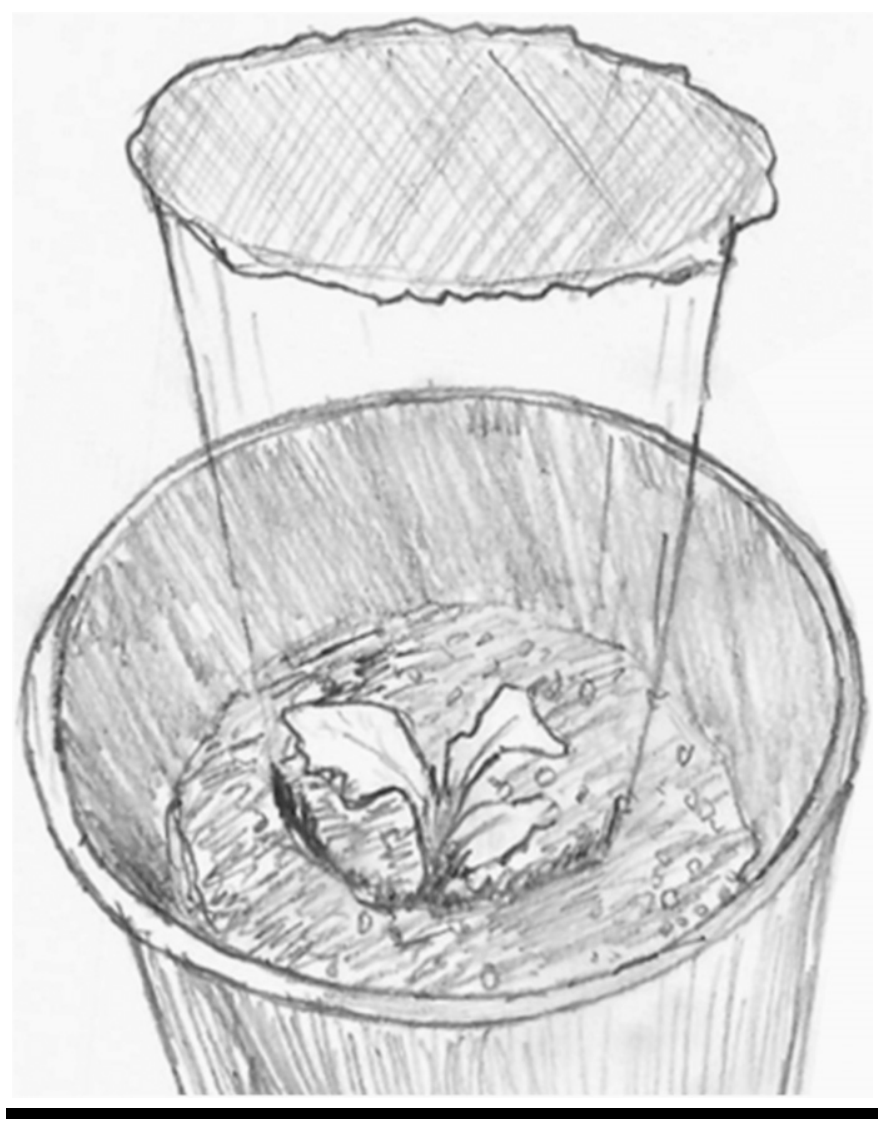

FIGURE 1

Diagram of a cage used to evaluate adult Lygus hesperus feeding and oviposition injury on a potted lettuce plant. stylet insertion on the surface of the lettuce plant was recorded. In addition, the number of lesions (Fig. 2A and B, shown with arrows) and eggs on the midrib of the lettuce leaves was recorded. To document signs of plant response to L. hesperus feeding or oviposition injury, plant parameters such as number of leaves, plant height $(\mathrm{cm})$, and fresh weight $(\mathrm{g})$ of above-ground structures of plants were recorded.

For analysis, numbers of lesions, feeding sites, eggs, and leaves as well as plant height and fresh weight were assessed by insect density for each exposure time. The data were analyzed to determine the effects of $L$. hesperus density, exposure time, and trial on injury symptoms and eggs as well as plant parameters such as plant height and fresh weight with interactions using the general linear model procedure of SAS (PROC GLM, SAS version 9.4, SAS Institute, Cary, NC). Data were log transformed $(\ln [x+1])$ to establish homogeneity of variance using the PROC Univariate procedure of SAS version 9.4 before analysis. When "trial" was included as a factor in the model, none of variables showed significant differences. Therefore, trial was not included in the final model. Because the exposure time $\times$ insect density interaction was significant for eggs and all lettuce plant parameters (Table 1), oneway ANOVA was performed by exposure time in PROC GLIMMIX, where insect density and replications were assigned as fixed and random effects, respectively, in the model. For each exposure time ( 2 or 7 days), both repeated trials were combined before analysis. The log link function and negative binomial distribution were used for these analyses. Least squares means were separated by pairwise $t$ tests $(P<0.05)$.

Correlations between numbers of lesions and numbers of eggs or feeding sites as well as plant parameters such as numbers of leaves, plant height, and fresh weight were analyzed using the PROC CORR procedure in SAS version 9.4. Before correlation analysis, dependent variable data collected for various $L$. hesperus density from two trials were pooled by exposure time.

\section{Effects of Adult Density and Time of Exposure on Injury}

The major injury symptom was lesions on the midrib (Fig. 2A to C). When $10 \mathrm{~L}$. hesperus adults fed for 7 days, lesions were found along the entire midrib region (Fig. 2C) or plants were completely destroyed (Fig. 2D). All eggs were recovered from the midrib, and most were found within lesions, as shown in Figure 2A. Although discolored spots were found on all regions of the leaf, most of them were observed on the midrib.

Effect of insect density was significant for the following variables: lesions, eggs, leaves, plant height, and fresh weight (Table 1). Likewise, the exposure time $\times$ insect density interaction was significant for leaves, plant height, and fresh weight. Effect of exposure time was only significant for fresh weight. Because the exposure time $\times$ insect density interaction was significant for most variables, the effect of insect density was further analyzed by exposure time after combining two similar repeated trials.

After exposing lettuce plants for 2 days, the number of lesions on leaves was significantly greater in $10 \mathrm{~L}$. hesperus than in 0,1 , and 5 L. hesperus treatments $\left(F_{4,36}=5.9 ; P<0.001\right.$; Fig. 3A). The number of feeding sites was significantly greater for 5 and $10 \mathrm{~L}$. hesperus treatments than for 0,1 , and $3 \mathrm{~L}$. hesperus treatments $\left(F_{4,36}=4.4 ; P=0.006 ;\right.$ Fig. $\left.3 \mathrm{~B}\right)$. A significantly greater number of eggs was found in the $10 \mathrm{~L}$. hesperus treatment than in the other $L$. hesperus treatments $\left(F_{4,36}=23.7 ; P<0.001\right.$; Fig. 3C). The number of leaves $\left(F_{4,36}=0.4 ; P=0.815\right)$, plant height $\left(F_{4,36}=0.7 ; P=\right.$ $0.569)$, and fresh weight $\left(F_{4,36}=1.1 ; P=0.359\right)$ of lettuce were not significantly different between treatments after 2 days exposure (Fig. 3D to F). 
After 7 days of exposure, numbers of lesions $\left(F_{4,28}=0.9 ; P=\right.$ $0.452)$, feeding sites $\left(F_{4,28}=0.1 ; P=0.980\right)$, and eggs $\left(F_{4,28}=1.9\right.$; $P=0.147)$ were not significantly different among $L$. hesperus density treatments (Fig. 4A to C). The number of leaves was significantly lower in the $10 \mathrm{~L}$. hesperus treatment than in the rest of the treatments $\left(F_{4,28}=2.8 ; P=0.045\right.$; Fig. 4D). Similarly, the plant height was significantly lower in the $10 \mathrm{~L}$. hesperus treatment than in the rest of the treatments $\left(F_{4,26}=6.7 ; P<0.001 ;\right.$ Fig. $\left.4 \mathrm{E}\right)$. The height of plants in 3 and 5 L. hesperus treatments was significantly lower compared with the insect-free control $(0 \mathrm{~L}$. hesperus $)$ treatment. The fresh weight of plants was significantly lower in $10 \mathrm{~L}$. hesperus treatment than in other treatments $\left(F_{4,27}=4.4 ; P=0.007\right.$; Fig. $\left.4 \mathrm{~F}\right)$.

The numbers of lesions and eggs $(r=0.41 ; P=0.003 ; n=50)$ as well as numbers of lesions and feeding sites $(r=0.39 ; P=0.004$;
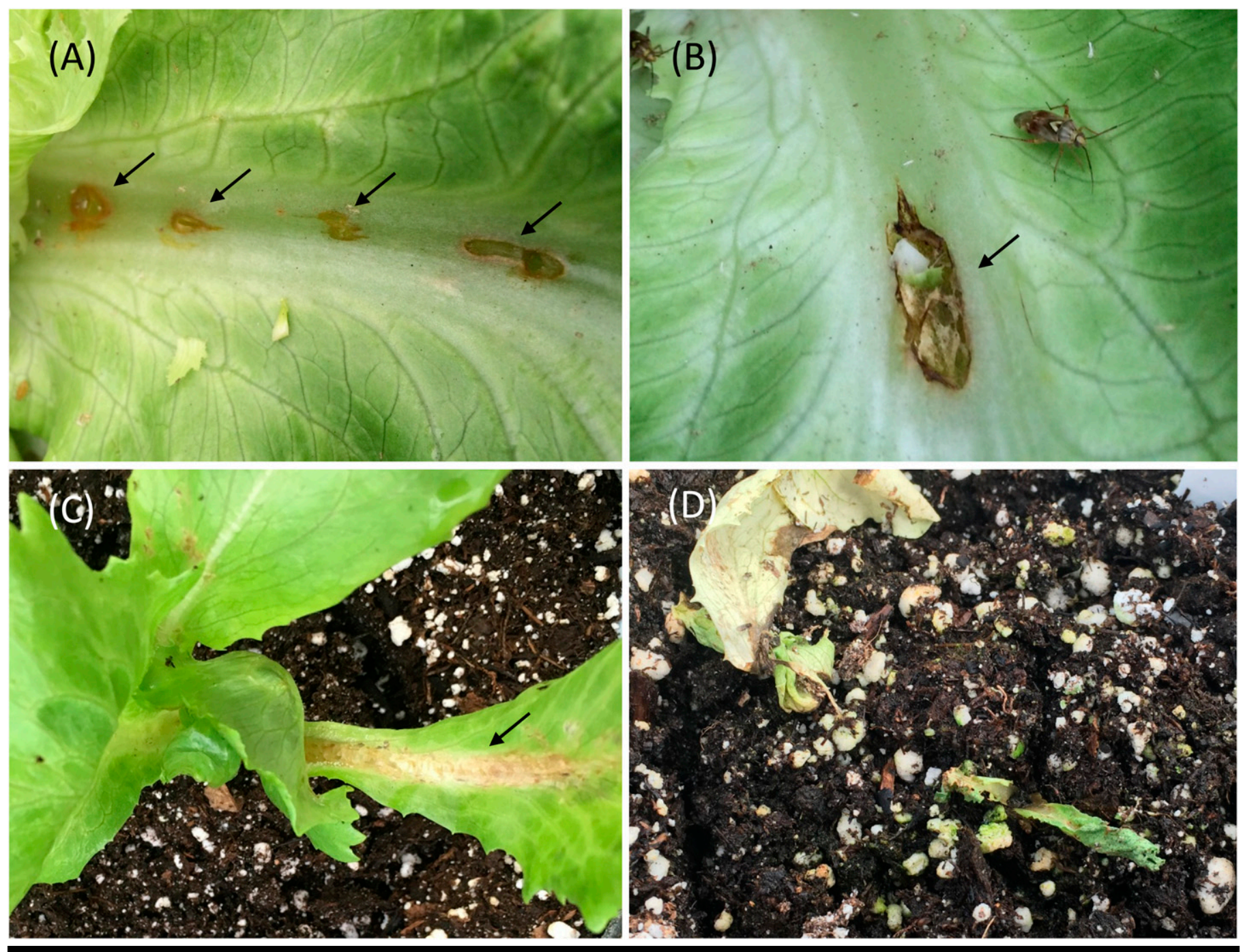

FIGURE 2

A and B, adult Lygus hesperus oviposition injury on the midrib of the leaf; $\mathbf{C}$, lesions developed after feeding on the midrib; and $\mathbf{D}$, plant destroyed after 7 days of exposure. The arrows indicate the oviposition injury or lesion developed after $L$. hesperus feeding.

TABLE 1

Summary of analysis of variance results for the model used to determine the effect of Lygus hesperus density and exposure time on feeding injury, egg deposition, and lettuce plant development ${ }^{\mathrm{a}}$

\begin{tabular}{|c|c|c|c|c|c|c|c|c|c|c|c|c|c|c|c|c|c|c|}
\hline \multirow[b]{2}{*}{ Factors } & \multicolumn{3}{|c|}{ Lesions } & \multicolumn{3}{|c|}{ Feeding sites } & \multicolumn{3}{|c|}{ Eggs } & \multicolumn{3}{|c|}{ Leaves } & \multicolumn{3}{|c|}{ Plant height } & \multicolumn{3}{|c|}{ Fresh weight } \\
\hline & df & $\boldsymbol{F}$ & $\boldsymbol{P}$ & df & $\boldsymbol{F}$ & $P$ & df & $\boldsymbol{F}$ & $\boldsymbol{P}$ & df & $\boldsymbol{F}$ & $\boldsymbol{P}$ & df & $\boldsymbol{F}$ & $\boldsymbol{P}$ & df & $\boldsymbol{F}$ & $\boldsymbol{P}$ \\
\hline ET & 1,87 & 2.6 & 0.111 & 1,87 & 0.4 & 0.543 & 1,87 & 1.5 & 0.228 & 1,87 & 0.9 & 0.343 & 1,85 & 0.7 & 0.402 & 1,86 & 5.6 & $0.021 *$ \\
\hline $\mathrm{ET} \times \mathrm{D}$ & 1,87 & 2.7 & 0.104 & 1,87 & 0.0 & 0.932 & 1,87 & 0.7 & 0.401 & 1,87 & 13.7 & $<0.001 *$ & 1,85 & 15.7 & $<0.001 *$ & 1,86 & 14.3 & $<0.001 *$ \\
\hline Repl. & 1,87 & 8.5 & $0.005^{*}$ & 1,87 & 0.2 & 0.689 & 1,87 & 2.6 & 0.114 & 1,87 & 0.2 & 0.667 & 1,85 & 0.5 & 0.477 & 1,86 & 0.1 & 0.748 \\
\hline
\end{tabular}

${ }^{\text {a }}$ Two exposure times ( 2 and 7 days) and five insect densities $(0,1,3,5$, and 10 L. hesperus adults per pot) were tested. Asterisk $(*)$ indicates significant difference $(P<0.05)$. ET = exposure time; $\mathrm{D}=$ insect density; and Repl. = replications. 
$n=50$ ) showed a significantly positive correlation at 2 days of exposure. However, the numbers of lesions and leaves $(r=0.12 ; P=$ $0.389 ; n=50)$, number of lesions and plant height $(r=-0.025$; $P=0.865 ; n=50)$, and number of lesions and fresh weight $(r=0.07$; $P=0.586 ; n=50$ ) did not show a significant association. After 7 days of exposure, the numbers of lesions and eggs showed a
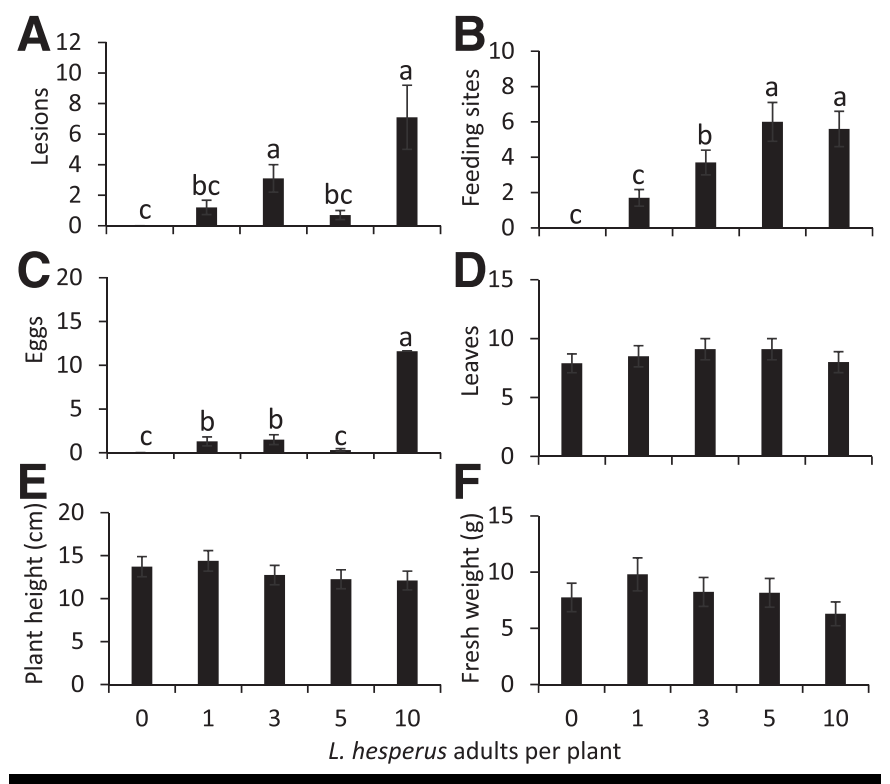

FIGURE 3

Least squares means ( \pm SEs) for numbers of $\mathbf{A}$, lesions; $\mathbf{B}$, feeding sites; $\mathbf{C}$, eggs; and $\mathbf{D}$, leaves; as well as $\mathbf{E}$, plant height $(\mathrm{cm})$; and $\mathbf{F}$, fresh weight $(\mathrm{g})$ of the lettuce after 2 days of adult $L$. hesperus exposure. Means followed by same letters between bars are not significantly different (pairwise $t$ test, $P \geq$ 0.05). Where no differences were observed, no letters are included.
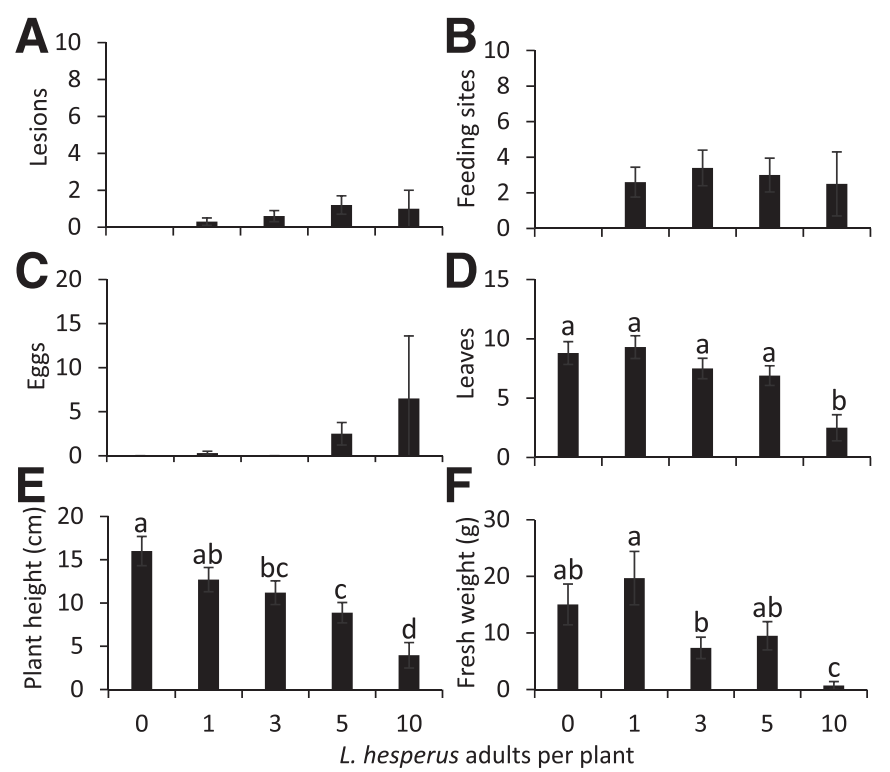

\section{FIGURE 4}

Least squares means ( \pm SEs) for numbers of $\mathbf{A}$, lesions; $\mathbf{B}$, feeding sites; $\mathbf{C}$, eggs; and $\mathbf{D}$, leaves; as well as $\mathbf{E}$, plant height $(\mathrm{cm})$; and $\mathbf{F}$, fresh weight $(\mathrm{g})$ of the lettuce after 7 days of adult $L$. hesperus exposure. Means followed by same letters between bars are not significantly different (pairwise $t$ test, $P \geq$ 0.05). Where no differences were observed, no letters are included. significantly positive correlation $(r=0.72 ; P<0.001 ; n=42)$, although numbers of lesions and feeding sites did not result in a significant association $(r=0.24 ; P=0.128 ; n=42)$. The number of lesions and plant height were positively correlated $(r=0.44 ; P=$ $0.004 ; n=40$ ), although number of lesions did not show a significant association with number of leaves $(r=-0.30 ; P=0.053$; $n=42)$ or fresh weight $(r=-0.08 ; P=0.600 ; n=41)$.

\section{Implications of the Results on Pest Management}

Results show that if heavy L. hesperus infestation (e.g., 10 adults per plant) lasted for up to a week, lesions developed rapidly and affected plant survival. Almost all plants exposed to the highest density of $L$. hesperus were killed after a week of exposure. During a shorter exposure period (e.g., up to 2 days), lettuce plants survived the injury, but the quality of lettuce was affected. In the central coast of California, lettuce is a 60- to 65-day crop from planting to harvest. It is $100 \%$ direct seeded, and seeds are planted at very high density and then thinned to stand at 21 to 27 days after planting. The injury or quality issues caused by $L$. hesperus infestation on lettuce might depend on whether the infestation occurred before or after the thinning stage. The results show that if the infestation occurred before thinning, the damage on lettuce plants could be absorbed as part of natural thinning. However, if $L$. hesperus invade at or after the thinning stage, damage can affect the normal plant development, causing quality issues or asynchronous lettuce stand at harvest. Presence of any feeding or oviposition damage closer to harvest can impact lettuce quality.

Under low to moderate levels of $L$. hesperus infestation, damage to lettuce can be minimized if timely control measures are administered. The current study indicates that injury symptoms caused by one or three $L$. hesperus adults were comparable to no adults for up to 2 days. However, if a moderate level of infestation ( $\sim 5$ adults) persists for a longer period (e.g., up to a week), the damage can affect crop quality. This suggests that although there is some room to administer control measures under moderate levels of infestation, it cannot be delayed beyond 2 days. If a moderate level of infestation lasts for about 7 days, data show that small lesions tend to coalesce into large lesions along the midrib.

In the current study, there is a strong association between numbers of lesions and eggs recovered from those lesions. L. hesperus females insert their eggs into the plant tissue (Wheeler 2001). This suggests that eggs deposited on the midrib are causing additional lesion injury. In addition to vascular bundles that run along the midrib, elongated collenchyma cells store water (Leroux 2012). The cell wall of collenchyma cells has irregular thickness (Leroux 2012), and L. hesperus prefers tender areas for oviposition (Alvarado-Rodriquez et al. 1986). Aggregated oviposition (Joseph et al. 2016) injures collenchyma tissue and vascular bundles in the mesophyll. Perhaps oviposition on the midrib damages tissue surrounding oviposition sites and, as the leaf expands, exacerbates lesion development (Fig. 2A and $\mathrm{B})$.

Results show that $L$. hesperus feeding injury can also lead to development of lesions on the midrib (Fig. 2C). There was a significant association between incidence of lesions and feeding sites. Adults survive by feeding on meristematic tissue in the crown or growing areas, where most photosynthates are translocated and concentrated (Joseph et al. 2016; Tingey and Pillemer 1977; Young 1986).

It is not clear if lettuce serves as a reproductive host for $L$. hesperus (Scott 1977), although young nymphs were found on the lettuce plants (S. V. Joseph, personal observation). The damage observed is probably related primarily to feeding and oviposition of invading adults. However, effects of immature L. hesperus developing on lettuce warrant further research. 
In the current study, feeding activity on the growing region of the lettuce was not observed, although previous studies have reported $L$. hesperus attacking apical buds of Douglas-fir (Pseudotsuga menziesii [Mirb.] Franco), causing bud abortion in nursery trees (Schowalter et al. 1986; South 1991), and causing necrotic dead tissue on floral buds of cotton (Gossypium hirsutum L.) (Mauney and Henneberry 1979). It is unclear if lettuce plants can support the energy needs of $L$. hesperus for prolonged periods. Typically, $L$. hesperus adults attack energy-rich plant parts such as seeds (Snodgrass 1998), which affects developing embryos (Scott 1970; Zalom et al. 2018). Perhaps on lettuce, transient L. hesperus adults seek shelter and water and may remain on lettuce plants until harvest.

In summary, the results provide insights into L. hesperus injury to lettuce. The impact of $L$. hesperus injury on lettuce quality and growth depends on the severity of $L$. hesperus infestation and duration of exposure. This suggests that infestations on lettuce closer to harvest can have severe economic consequences if not addressed with timely management measures, such as insecticide sprays. Pyrethroid and neonicotinoid insecticides are effective against $L$. hesperus (Joseph et al. 2017; Zalom et al. 2018). Pyrethrins can provide adult suppression in organic production systems. Economic losses related to $L$. hesperus injury can be driven by market value. When market value is high, the affected wrapping leaves are trimmed off during harvest, and the rest of the lettuce is marketed. If $L$. hesperus or its damage are detected during preharvest inspection, it could lead to rejection of an entire field. This can result in a $\sim$ US\$75,000 loss for a typical 6-ha lettuce field. Based on the results, timely management is warranted once $L$. hesperus adults are identified on lettuce to reduce losses associated with injury.

\section{Acknowledgments}

The author appreciates the help provided by E. Cassias and A. Valdez in setup of the trials and thanks Dow AgroScience, DuPont, and Bayer for their funding support.

\section{Literature Cited}

Alvarado-Rodriquez, B., Leigh, T. F., and Foster, K. W. 1986. Oviposition site preference of Lygus hesperus (Hemiptera: Miridae) on common bean in relation to bean age and genotype. J. Econ. Entomol. 79:1069-1072.

Backus, E. A., Serrano, M. S., and Ranger, C. M. 2005. Mechanisms of hopperburn: An overview of insect taxonomy, behavior, and physiology. Annu. Rev. Entomol. 50:125-151.

Fye, R. E. 1984. Damage to vegetable and forage seedlings by overwintering Lygus hesperus (Heteroptera: Miridae) adults. J. Econ. Entomol. 77: 1141-1143.
Hill, L. L. 1932. Protection of celery from tarnished plant bug injury. J. Econ. EntomoI. 25:671-678.

Hill, L. L. 1933. Further studies of tarnished plant bug injury to celery. J. Econ. EntomoI. 26:148-150.

Jacobson, R. J. 2002. Lygus rugulipennis (Het. Miridae): Options for integrated control in glasshouse-grown cucumbers. OILB/SROP Bull. 25:111-114.

Joseph, S. V., Ahedo, R., and de la Fuente, M. 2016. Characterization of Lygus hesperus (Hemiptera: Miridae) feeding and oviposition injury on celery seedlings. Plant Health Prog. 17:101-105.

Joseph, S. V., Martin, T., Steinmann, K., and Kosina, P. 2017. Outlook of pyrethroid insecticides for pest management in the Salinas Valley of California. J. Integr. Pest Manag. 8:6.

Leroux, O. 2012. Collenchyma: A versatile mechanical tissue with dynamic cell walls. Ann. Bot. 110:1083-1098.

Mauney, J. R., and Henneberry, T. J. 1979. Identification of damage symptoms and patterns of feeding of plant bugs in cotton. J. Econ. Entomol. 72:496-501.

McPherson, J. E., ed. 2018. Invasive Stink Bugs and Related Species (Pentatomoidea): Biology, Higher Systematics, Semiochemistry, and Management. CRC Press, Boca Raton, FL.

Miles, P. W. 1972. The saliva of Hemiptera. Adv. Insect Physiol. 9:183-255.

Monterey County Agricultural Commissioner. 2017. Monterey County Crop Report, 2017. Office of the Agricultural Commissioner, Salinas, CA. http:// www.co.monterey.ca.us/Home/ShowDocument?id=65737

Natwick, E. T., Joseph, S. V., and Dara, S. K. 2017. Lygus bug (western tarnished plant bug). UC IPM Pest Management Guidelines: Lettuce. UC ANR Publication 3450. https://www2.ipm.ucanr.edu/agriculture/lettuce/lygus-bugwestern-tarnished-plant-bug/

Schowalter, T. D., Overhulser, D. L., Kanaski, A., Stein, J. D., and Sexton, J. 1986. Lygus hesperus as an agent of apical bud abortion in Douglas-fir nurseries in western Oregon. New For. 1:5-15.

Scott, D. R. 1970. Feeding of Lygus bugs (Hemiptera: Miridae) on developing carrot and bean seed: Increased growth and yields of plants grown from that seed. Ann. Entomol. Soc. Am. 63:1604-1608.

Scott, D. R. 1977. An annotated listing of host plants of Lygus hesperus Knight. Bull. Entomol. Soc. Am. 23:19-22.

Snodgrass, G. L. 1998. Distribution of the tarnished plant bug (Heteroptera: Miridae) within cotton plants. Environ. Entomol. 27:1089-1093.

South, D. B. 1991. Lygus bugs: A worldwide problem in conifer nurseries. Pages 215-222 in: Proceedings of the First IUFRO Workshop on Diseases and Insects in Forest Nurseries, Victoria, BC. Forestry Canada, Information Report BC-X-331.

Strong, F. E. 1970. Physiology of injury caused by Lygus hesperus. J. Econ. Entomol. 63:808-814.

Tingey, W. M., and Pillemer, E. A. 1977. Lygus bugs: Crop resistance and physiological nature of feeding injury. Bull. Entomol. Soc. Am. 23:277-287.

Varis, A. L. 1978. Lygus rugulipennis (Heteroptera, Miridae) damaging greenhouse cucumbers. Ann. Entomol. Fenn. 44:72.

Wheeler, A. G., Jr. 2001. Page 507 in: Biology of the Plant Bugs (Hemiptera: Miridae): Pests, Predators, Opportunists. Cornell University Press, Ithaca, NY.

Young, O. P. 1986. Host plants of the tarnished plant bug, Lygus lineolaris (Heteroptera: Miridae). Ann. Entomol. Soc. Am. 79:747-762.

Zalom, F. G., Bolda, M. P., Dara, S. K., and Joseph, S. V. 2018. Lygus bug. UC IPM Pest Management Guidelines: Strawberry. UC ANR Publication 3468. https://www2.ipm.ucanr.edu/agriculture/strawberry/lygus-bug/. 